\begin{tabular}{l|l} 
REVISTA & $\begin{array}{l}\text { Revista Educación } \\
\text { ISSN: 0379-7082 } \\
\text { ISSN: 2215-2644 } \\
\text { revedu@gmail.com } \\
\text { Universidad de Costa Rica } \\
\text { Costa Rica }\end{array}$
\end{tabular}

\title{
Formación docente en educación secundaria en la Universidad de Costa Rica
}

\author{
Chaves-Salas, Ana Lupita; Castro-Bonilla, Julieta \\ Formación docente en educación secundaria en la Universidad de Costa Rica \\ Revista Educación, vol. 41, núm. 2, 2017 \\ Universidad de Costa Rica, Costa Rica \\ Disponible en: http://www.redalyc.org/articulo.oa?id=44051357014
}

Esta obra está bajo una Licencia Creative Commons Atribución-NoComercial-SinDerivar 3.0 Internacional. 


\section{Formación docente en educación secundaria en la Universidad de Costa Rica}

\section{Teacher training in High School Education at the University of Costa Rica}

Ana Lupita Chaves-Salas [1]

Redalyc: http://www.redalyc.org/articulo.oa?id=44051357014

Universidad de Costa Rica, Costa Rica

ana.chaves@ucr.ac.cr

Julieta Castro-Bonilla [2]

Universidad de Costa Rica, Costa Rica

julietacastrob@gmail.com

\section{Resumen:}

El propósito de este artículo es presentar una reseña histórica de la formación docente en educación secundaria en la Universidad de Costa Rica, desde su creación en 1941 hasta la actualidad. Se describen los planes de estudio, las áreas de formación, el creditaje y el modelo de gestión curricular y administrativa de las carreras. La formación del profesorado se ha caracterizado por ser integral e interdisciplinaria, hasta el año 2016 cuando la Vicerrectoría de Docencia aprueba una carrera disciplinar con enfoque tecnocrático contrario a las tendencias actuales sobre formación de docentes. El artículo surge de una investigación descriptiva-comparativa sobre modelos de formación de docentes en educación secundaria en cinco países: Alemania, Cuba, Finlandia, Singapur y Costa Rica. Las técnicas utilizadas fueron el análisis de documentos y la entrevista a informantes clave. Descriptores: Formación inicial docente, educación secundaria, planes de estudio, educación superior.

PALABRAS ClaVE: Formación inicial docente, educación secundaria, planes de estudio, educación superior.

\section{Abstract:}

The purpose of this article is to present a historical review of teacher education in higher education at the University of Costa Rica, from its creation in 1941 until today. Curricula, training areas, credits, and the curricular and administrative management models of majors are described. The training of teachers has been characterized as comprehensive and interdisciplinary until the year 2016 when the Subdirector of Teaching approved a disciplinary major with a technocratic approach contrary to current trends in teacher education training. The article deals with a descriptive-comparative research on models of teacher education in secondary education in the following five countries: Germany, Cuba, Finland, Singapore, and Costa Rica. The techniques used for this research paper were the analysis of documents and interviews to key informants.

KEYWORDS: initial teacher training, secondary education, curriculum, higher education.

\section{NotAS DE AUTOR}

[1] Doctora en Educación. Magister en Educación de Adultos. Bachiller y Licenciada en Educación Preescolar. Actualmente es representante de la Universidad de Costa Rica en el Consejo Superior de Educación. Fue Decana de Facultad de Educación de la Universidad de Costa Rica y directora del Programa de Posgrado Académico en Educación. Es investigadora del Instituto de Investigación en Educación de la UCR y fue su directora del 2001 al 2008. Docente de la carrera de Bachilllerato y Licenciatura en Educación Preescolar de la Escuela de Formación Docente. Directora de tesis de grado y posgrado. Participa como ponente en congresos nacionales e internacionales. Es miembro del Consejo Editorial de la Revista en español de la International Reading Association y de la Revista de Educación de la Universidad Central de Venezuela. Es autora de libros, capítulos de libros y numerosos artículos en revistas nacionales y de otros países sobre una diversidad de temas: educación preescolar, procesos iniciales de lectoescritura, investigación-acción en el aula, formación docente, escuelas unidocentes, docentes que han destacado, evaluación en secundaria, entre otros. Coordina proyectos de extensión dirigidos a docentes en zonas urbanas y rurales.

[2] Master of Science in Education State University of New York at Buffalo, New York. USA. Profesora y Bachiller en la Enseñanza de Artes Plásticas. Facultad de Educación, Universidad de Costa Rica. Bachiller en Artes Plásticas y Licenciada en Bellas Artes con especialidad en Pintura. Facultad de Bellas Artes, Universidad de Costa Rica. Participación en la formación de estudiantes de educación preescolar, primaria y secundaria en instituciones del Estado y privadas. Impartió los cursos de Pedagogía, Didáctica, Artes Plásticas en la Educación Preescolar y Primaria, así como Metodología en las Artes Plásticas. Supervisa la práctica de estudiantes en el área de la enseñanza de las Artes Plásticas. En los últimos 15 años forma parte del Comité Editorial de la Revista “Actualidades Investigativas en Educación”, del Instituto de Investigación en Educación (INIE) y realiza investigación enfatizando la integración curricular de las artes a todo proceso educativo. Los resultados de esas investigaciones se convierten en actividades de acción social por medio de talleres en diversas zonas e instituciones del país. Ha escrito artículos científicos y libros de texto que favorecen la integración del arte al currículo escolar y sirven de material didáctico para que la población docente lleve a la práctica metodologías innovadoras en el contexto escolar. En la actualidad participa en actividades de investigación y de acción social en el INIE, así como con en organismos no gubernamentales. 


\section{INTRODUCCIÓN}

Este artículo presenta los resultados de un estudio que se realizó sobre la formación inicial docente en educación secundaria en la Universidad de Costa Rica (UCR). Se hace un breve recorrido histórico desde su creación en 1941, se describen los planes de estudio actuales, áreas de formación, cursos que se ofrecen, créditos, titulación y el modelo gestión administrativa y curricular mediante comisiones integradas por representantes de la Escuela de Formación Docente, de las Unidades Académicas que ofrecen las disciplinas de la especialidad y por las respectivas representaciones estudiantiles, modelo de gestión que ha generado algunas tensiones a lo largo de los años.

La investigación que da origen a este artículo tiene como objetivo general analizar modelos de formación inicial de docentes de ese nivel educativo en países con altos índices de desarrollo humano y compararlos con el que ofrece la Universidad de Costa Rica, con el propósito de diseñar una propuesta para que sea discutida en el seno de las Unidades Académicas que participan en la formación de profesionales en educación de ese nivel educativo. Los resultados de este estudio se han divulgado y publicado mediante diferentes actividades académicas y artículos.

La investigación emergió del Plan Estratégico 2011- 2015 de la Facultad de Educación (FE) de la Universidad de Costa Rica (UCR), que definió como programa prioritario del eje de docencia denominado: Autoevaluación de los planes de estudio para el mejoramiento y la acreditación.

En el caso de las carreras de educación secundaria, un grupo de docentes investigadoras de la Escuela de Formación Docente y del Instituto de Investigación en Educación, decidió realizar la investigación y se dieron a la tarea de analizar los modelos sobre formación docente que ofrecen los siguientes países: Alemania, Cuba, Finlandia, Singapur y Costa Rica, mediante un estudio descriptivo comparativo, para lo cual se realizó una exhaustiva revisión bibliográfica nacional e internacional.

$\mathrm{El}$ artículo concluye con algunas reflexiones sobre la formación docente que se ofrece en la UCR.

\section{Metodología}

La investigación es de tipo descriptiva-comparativa, en la que se utilizaron técnicas cualitativas: análisis de documentos y entrevistas. Para el análisis de los planes de formación docente de los países mencionados, se utilizó el método de educación comparada para determinar las semejanzas y diferencias de dichos planes con base en los indicadores que se definieron para este estudio a partir de los objetivos de la investigación y la bibliografía revisada. Como se indicó, este artículo únicamente se aboca a conocer la formación de docentes en educación secundaria de la Universidad de Costa Rica.

Las etapas de la investigación se describen seguidamente:

1. Revisión bibliográfica.

2. Selección de países para el analizar el modelo de formación docente en educación secundaria.

3. Definición de técnicas, elaboración y validación de instrumentos.

4. Aplicación de técnicas e instrumentos:

- Análisis documental de los planes de estudio.

- Aplicación de entrevistas.

5. Análisis de la información

- Definición de categorías de análisis.

- Análisis de la información mediante matrices y la triangulación de la información

Seguidamente se presentan los resultados obtenidos sobre la formación de docentes en educación secundaria en la UCR. 


\section{Formación docente en educación secundaria en la Universidad de Costa Rica (1941-2015)}

La formación docente en Costa Rica se ha ido transformando con el transcurso del tiempo. De acuerdo con Dengo (2013), a principios del Siglo XX gran cantidad de docentes de educación secundaria eran del extranjero, con estudios que enfatizaban enfoques técnicos-academicistas, otra parte era costarricense que habían obtenido becas de estudio para formarse en Chile y se les otorgaba el título de "Profesor de Estado", otros eran docentes con cinco años de experiencia en educación secundaria que se sometían a un examen en la especialidad que consistía en ofrecer una exposición de su materia y la elaboración de una tesina en su área de estudio, la cual exponían ante un tribunal constituido por personal directivo de los cinco colegios públicos que existían en el país.

En 1940, mediante el Decreto N.³62 del Poder Ejecutivo, la Sección Normal, encargada de la formación docente en educación primaria, pasa a denominarse Escuela de Pedagogía en la Universidad de Costa Rica y el requisito para ingresar era contar con el título de Bachiller en Ciencias y Letras de la Educación Secundaria. Este hecho es relevante debido a que la formación de docentes de educación primaria deja de ser exclusiva del Estado costarricense al delegarse parte de esta formación en la UCR, institución autónoma.

En 1941, la Escuela de Letras y Filosofía y la Escuela de Ciencias de la Universidad de Costa Rica inician la formación de profesionales en esa área, así como de docentes de educación secundaria con énfasis en Ciencias y Letras. Ingresaban estudiantes con Bachillerato en Ciencias y Letras o maestros y maestras que habían egresado de la Escuela Normal. "El título que otorgaban fue el de 'Licenciados en Letras y Filosofía' y 'Licenciado en Ciencias', con la mención de la especialidad de estudios: por ejemplo: 'Filosofía' o 'Historia', etc." (Dengo, 2013, p. 248). Estos planes de estudio contenían las siguientes materias: metodología en el área de la disciplina, práctica y psicología educativa, además de los cursos propios de la disciplina. En cuanto a la formación profesional, la proporción de los cursos de pedagogía era reducida.

En 1954, el rector Rodrigo Facio propone una nueva organización en la UCR, con el propósito de evitar la fragmentación y promover la integración académica; de esta manera, se crea el Departamento de Estudios Generales que ofrecería una formación inicial a toda la población estudiantil que ingresara a la UCR. Se funda la Facultad de Ciencias y Letras, que reúne las Escuelas de Letras y Filosofía y la Escuela de Ciencia (Dengo, 2013).

La Facultad de Ciencias y Letras brindaría la formación disciplinar a estudiantes que seguirían la carrera de profesorado en educación secundaria, y la nueva Facultad de Educación ofrecería la formación pedagógica y sería la encargada de otorgar los títulos de Profesor de Segunda Enseñanza. La Reforma Universitaria entra en vigencia en 1957 y después de la Reforma de la Enseñanza Media (1964-1965), impulsada por el Ministerio de Educación Pública, se cambia el nombre por Título por Profesor de Enseñanza Media en -el área de formación correspondiente (Dengo, 2013). Este modelo de formación de docentes entró en crisis, debido a que la Facultad de Ciencias y Letras quiso recobrar la formación de docentes, por tal razón se creó una Comisión Coordinadora del Programa de Formación de Profesores de Enseñanza Media, se estudió la situación y las carreras se mantuvieron en la Facultad de Educación (González, 2013).

La Reforma Universitaria de 1957 fue visionaria, pues imprime una formación integral y humanista a las personas graduadas de la Universidad de Costa Rica (Polanco,1990), más que formar en una disciplina de manera fragmentada se busca una formación holista-humanista en los futuros profesionales que servirán al país.

En ese entonces la Facultad de Educación tenía como decana a la señora Emma Gamboa, quien poseía un Doctorado en Filosofía con énfasis en Educación obtenido en la Universidad del Estado de Ohio, Estados Unidos, institución universitaria que promovía la "Escuela Activa" de John Dewey cuyo lema era "educar para las sociedades libres”. Al respecto Dengo (2013, p. 252) indica: 
...lo que fue más característico en su filosofía educacional fue su convicción democrática, su profunda fe en la capacidad transformadora de la educación, y por tanto de los educadores, para influir en los procesos de cambio social y, asimismo, en la formación de valores de libertad, de dignidad, de derechos humanos en la colectividad costarricense."

Dengo (2013) opina que esta filosofía influyó en la formación de docentes en la Facultad de Educación de la UCR.

A partir de la Reforma Universitaria de 1957 y acorde con la Ley Fundamental de Educación (Asamblea Legislativa de la República de Costa Rica, 1957), se fortalece el área pedagógica en la formación de docentes y se incluyen los siguientes cursos: Historia de la Educación (2 horas), Filosofía de la Educación ( 2 horas), Sociología de la Educación (2 horas), Educación secundaria (Fundamentos y Programas) (2 horas), Psicología del adolescente ( 2 horas), Métodos y práctica docente en el área de la especialización (6 horas), Psicología de la educación (2 horas) y Métodos y práctica docente en el área de la especialización (6 horas), (UCR, 1960).

Durante esa época, había un faltante de docentes de educación secundaria con título, lo cual es ratificado por el Ministerio de Educación Pública al emitir en el año 1958 un documento, en el que indica que se "carece de personal docente especialmente preparado para cumplir su función a nivel de enseñanza media. La situación en marzo de 1957 era la siguiente: de un total de 1.067 profesores (100\%), el 35\% corresponde al grupo de aspirantes; el 49\% a los profesores del grupo B; el resto, al grupo A. En el grupo B, que constituye casi el 50\% del profesorado, se encuentran profesores que carecen de preparación ya sea en la especialidad, o en educación” (Ministerio de Educación Pública, 1958, p. 52). Es decir, a mediados del Siglo XX solo un pequeño grupo de docentes había consolidado la formación universitaria en educación secundaria.

En mayo de 1967, se crea la Escuela Normal Superior como una sección de la Escuela Normal de Heredia, la cual inició labores en 1968 y las concluyó en 1973 debido a que pasó a formar parte de la Universidad Nacional (UNA), la cual se fundó ese mismo año. Esta universidad absorbe a otras instituciones formadoras de docentes del Ministerio de Educación Pública (MEP): Escuela Normal de Guanacaste, Escuela Normal de San Ramón, Escuela Normal de Pérez Zeledón y Centro de Capacitación (Dengo, 2013).

Con el transcurso del tiempo, la Facultad de Educación de la UCR fue ofreciendo otras especialidades para la educación media como Educación Física, Psicología, entre otras.

Con el inicio de la regionalización de la UCR en 1968, en las Sedes en San Ramón de Alajuela y de Liberia, se inició la formación de docentes de acuerdo con las necesidades de las zonas.

Posteriormente, como producto del Tercer Congreso Universitario en 1972, surgen cambios en la organización de la UCR, de tal manera que en 1977 "la Facultad de Educación se integra en tres escuelas: Administración Educativa, Orientación y Educación Especial y Formación Docente, y se establece el bachillerato en la enseñanza secundaria para cada una de las áreas de formación académica con 135 créditos, de los cuales $42(31 \%)$ corresponden a la formación pedagógica, lo que implicó el fortalecimiento en esta área, ya que desde 1967, únicamente 28 créditos (25\%) se destinaban al área antes mencionada” (Dengo, 2013, p. 255).

Años después se crean otras Unidades Académicas en la Facultad de Educación: la Escuela de Educación Física y la Escuela de Bibliotecología y Ciencias de la Información.

La gestión administrativa y curricular de las carreras de educación secundaria en la Universidad de Costa Rica se regula en el año 1989 mediante la Resolución 4379-89 y también en ese año se crea el Departamento de Educación Secundaria, en la Escuela de Formación Docente. Esta Resolución instaura las Comisiones Compartidas (CC) e indica que estas carreras "se ofrecerán bajo la responsabilidad conjunta de la Escuela de Formación Docente y cada una de las siguientes Escuelas: Artes Plásticas; Artes Musicales; Lenguas Modernas; Filología, Lingüística y Literatura; Psicología; Historia y Geografía; Filosofía; Biología; Física; Química y Matemática" (UCR, 1989, p. 1).

Las carreras de educación secundaria de la UCR tienen su padrón estudiantil en la Escuela de Formación Docente de la Facultad de Educación, debido a que su objeto de estudio es la formación docente y el eje transversal es la pedagogía como la ciencia que estudia los procesos educativos. 
En la Resolución 4379-89 se crea también el Comité de Directores de las Escuelas que participan en la formación de docentes, el cual será presidido de manera permanente por el director o directora de la Escuela de Formación Docente y se le definen las siguientes funciones: instalar las comisiones compartidas, servir de vínculo entre la UCR, el MEP, el Consejo Superior de Educación (CSE), entre otros entes externos vinculados con la educación y su mejoramiento; proponer políticas para la formación de docentes, reunir y asesorar a las CC y evaluar su desempeño.

La resolución asigna a las Comisiones Compartidas las siguientes funciones: revisar y actualizar el perfil profesional de las carreras, analizar las propuestas de modificación a los planes de estudio y hacerlo de conocimiento de las unidades académicas que participan en su implementación, coordinar el proceso de admisión a las carreras, mantener los expedientes estudiantiles actualizados, estudiar solicitudes de otras sedes para desconcentrar las carreras, entre otras. También indica que la carga académica que se le asigna a la persona que coordine la Comisión Compartida será por mutuo acuerdo de las unidades académicas que intervienen en la carrera, de acuerdo con el número de estudiantes inscritos (UCR, 1989).

Para llevar a cabo las funciones señaladas, las Comisiones Compartidas estarán integradas por "dos profesores de cada unidad académica (4) y un representante estudiantil inscrito y activo de la carrera." (UCR, 1989 , p. 2), además se indica que son miembros ex oficio de estas Comisiones, con voz y voto, las personas que ocupen los puestos de la Dirección de las Unidades Académicas. La integración de cada Comisión tiene como propósito ofrecer equidad en la toma de decisiones de acuerdo con el espíritu democrático que distingue a la UCR y que se plasma en su Estatuto Orgánico:

Artículo 4: Son principios orientadores del quehacer de la Universidad:

b. Excelencia académica e igualdad de oportunidades: Velar por la excelencia académica de los programas que ofrezca, en un plano de igualdad de oportunidades y sin discriminación de ninguna especie

Esta Resolución fue firmada por la Vicerrectora de Docencia de ese entonces, la Licda. Elizabeth Odio Benítez.

Las carreras en la enseñanza de educación secundaria que ofrece la UCR en 1989 son las siguientes: Inglés, Francés, Estudios Sociales, Ciencias Naturales, Filosofía, Castellano y Literatura, Artes Plásticas, Matemática, Música y Psicología.

En algunos casos las Comisiones Compartidas son integradas por tres, cuatro o cinco unidades académicas, como en el caso de la Enseñanza de las Ciencias en la que participan las Escuelas de Química, Física, Biología, Geología y Formación Docente, por lo que estas Comisiones están integradas hasta por diez personas, y algunas veces se hace difícil conciliar agendas para las reuniones, por tal razón en el año 2000, siendo Vicerrector de Docencia el Dr. Ramiro Barrantes, se redacta una adición a la Resolución 4379-89, en la cual se designa un representante y un suplente para cada Escuela, a excepción de la Escuela de Formación Docente que continuaría con dos representantes (UCR, 2000).

La gestión administrativa y curricular de esas carreras mediante Comisiones Compartidas ha sido poco ágil para llevar a cabo todas las gestiones que requiere un plan de estudio, así como para realizar modificaciones, innovaciones, procesos de autoevaluación y acreditación, debido a que todo cambio debe contar con la aprobación de las unidades académicas que participan en la formación de ese futuro personal docente. Cabe señalar que algunas comisiones enfrentan conflictos internos que no les permiten ejecutar con eficiencia su labor.

En 1990 se reestructuran los planes de estudio de formación de docentes de educación secundaria, algunos de los cambios se relacionan con el perfil profesional, la práctica docente se reduce a un semestre, se crea una salida lateral para otorgar el Profesorado con tres años de estudios universitarios, ante la demanda de docentes de educación secundaria por parte del MEP, entre otras transformaciones que se hacían necesarias en ese momento. 
A mediados de la década de los 90, la facultad de Educación evidenció la necesidad de autoevaluar las carreras que ofrecían las diferentes unidades académicas para su mejoramiento y acreditación; sin embargo, estos procesos no tuvieron el éxito esperado

Para analizar el quehacer de la Facultad de Educación en docencia, investigación y acción social, durante los meses de noviembre y diciembre del 2010, se diseña el Plan Estratégico de la Facultad de Educación. Este Plan se elabora de manera participativa, con la contribución del personal docente y administrativo, estudiantes; representantes de las Vicerrectorías de la UCR, así como autoridades del MEP, del Estado de la Educación, la Estrategia hacia el Siglo XXI, el SINAES, los gremios, el Colegio de Licenciados y Profesores, entre otros. Entre los programas prioritarios que surgen del Plan Estratégico, se define la Autoevaluación permanente de las carreras con miras al mejoramiento y a la acreditación, con el propósito de ofrecer a la educación costarricense propuestas académicas innovadoras acordes con las nuevas demandas educativas del mundo actual (Chaves, 2012a). En consecuencia, se fortalecen los procesos de autoevaluación para la acreditación de Planes de Estudio que ya se encontraban en proceso en la Escuela de Formación Docente, en la Escuela de Orientación y Educación Especial; de reacreditación en la Escuela de Educación Física y Deporte, y se inician en la Escuela de Administración Educativa y Escuela de Bibliotecología y Ciencias de la Información (Chaves, 2011). Asimismo, el Decanato y la Dirección de la Escuela de Formación Docente, en coordinación con el Sistema Nacional de Acreditación (SINAES), diseñan una propuesta de autoevaluación con miras a la acreditación: Modelo de Acreditación por Conglomerados de las Carreras de Enseñanza Secundaria de la Escuela de Formación Docente de la Universidad de Costa Rica (Chaves, 2011). Sin embargo, esta propuesta no fue implementada en esas carreras. En el año 2012, el Centro de Evaluación Académica (CEA) de la Universidad de Costa Rica (UCR) presenta otro modelo de autoevaluación con miras a la acreditación y al mejoramiento de esos planes de estudio (Chaves, 2012a).

Paralelamente, durante el 2011, la Vicerrectoría de Docencia (VD), a cargo de la Dra. Libia Herrero, propuso analizar el modelo de Comisión Compartida de las diferentes carreras de la enseñanza secundaria. Esta labor se realizó, en un primer momento, en el seno de cada una de las diez Comisiones Compartidas responsables de la formación de docentes para las respectivas asignaturas de la educación secundaria, las cuales emitieron propuestas relevantes para su reorganización. En un segundo momento, se hacen reuniones con el propósito de que cada Comisión Compartida exponga sus criterios a las Direcciones de las Unidades Académicas y a las Decanaturas y a representantes de la Vicerrectoría de Docencia sobre la gestión administrativa y curricular de las carreras de educación secundaria. Se realizan diez reuniones, una por Comisión Compartida. La mayoría de estas firman los siguientes acuerdos: 1- autoevaluar las carreras a partir de los resultados de investigación científica para el rediseño de los planes de estudio con miras al mejoramiento o a la acreditación, 2- reestructurar y fortalecer las Comisiones Compartidas con el fin de asignarles un mayor nivel de autonomía y promover una gestión ágil, y 3-adjudicar mayores recursos presupuestarios y humanos para el trabajo de estas Comisiones, de manera que puedan desarrollar proyectos conjuntos y cumplir con lo firmado (Chaves, 2012b).

El propósito de la Vicerrectoría de Docencia era elaborar una nueva resolución para la administración de las carreras de educación secundaria con base en los acuerdos firmados por las personas que formaban parte de las Comisiones Compartidas, las Direcciones de Unidades Académicas, Decanatos, Vicerrectoría de Docencia y representantes de esa instancia, por lo que emite la Resolución R-8782- 12 el 23 de febrero del 2012 (UCR, 2012a), la cual es anulada por la Rectoría mediante la Resolución R-2616-2012 el 16 de mayo de ese mismo año (UCR, 2012b), como respuesta a un recurso de revocatoria presentado por la Escuela de Formación Docente con apoyo del Decanato de la Facultad de Educación. La Resolución de la Vicerrectoría, entre otros cambios, traslada la formación de docentes en la enseñanza de la matemática y en la enseñanza de la música, a la Escuela de Matemática y a la Escuela de Música, respectivamente, en cuyo proceso formativo no tendría injerencia la Facultad de Educación. La Escuela de Formación Docente y el Decanato de la Facultad indican que la Resolución emitida carecía de fundamento científico-pedagógico, pues no tomaba en cuenta 
las corrientes actuales en cuanto a la formación de docentes, no promovía la interdisciplinaridad de manera generalizada, y no reflejaba la mayoría de los acuerdos firmados de manera conjunta por la misma Vicerrectora de Docencia y las Unidades Académicas. Además, contenía vicios de nulidad de acuerdo con la normativa universitaria, al no responder al modelo democrático y participativo de Universidad que establece el Estatuto Orgánico de la UCR.

En de 2012, siendo Vicerrector de Docencia el Dr. Bernal Herrera, amplía la Resolución VD--8873-2012 del 17 de octubre, y emite la Resolución 4379-89 (UCR, 1989) que regula la gestión curricular y administrativa de las carreras de educación secundaria. Se mantienen y amplían las funciones de las Comisiones Compartidas y las denomina Comisiones de Carreras de la Enseñanza, a las que les asigna, entre otras, las siguientes funciones: seleccionar al personal docente que ofrecerá los cursos de las carreras de una terna preparada por las Unidades Académicas, labor que se llevará a cabo cada ciclo lectivo; realizar procesos de inducción, seguimiento y supervisión al profesorado y al estudiantado, así como evaluar al personal docente; estas Comisiones deben llevar a cabo los procesos relacionados con el diseño, elaboración y ejecución del trabajo final de graduación del estudiantado. También indica que, si la Comisión respectiva no llega a acuerdos en relación con las modificaciones a los planes de estudio, la directora del Centro de Evaluación Académica podrá participar con voz y voto para determinar los procesos y cambios que correspondan. Esa Resolución cambia la conformación de las Comisiones de las Carreras de la Enseñanza, dejando en desventaja a la Escuela de Formación Docente, al asignarle únicamente dos representantes y tres a las otras cadémicas (en la Resolución de 1989, la representación era equitativa, dos representantes por escuela) y, en el caso de carreras que participan más de una del área disciplinar, se les fija dos representantes. Esta Resolución muestra debilidad en el aspecto académico, puesto que ignora que el objeto de estudio de estas carreras es la formación docente y su eje transversal la pedagogía, ciencia de responsabilidad de la Escuela de Formación Docente y de la Facultad de Educación, y es contraria a los principios democráticos establecidos en el Estatuto Orgánico de la Universidad de Costa Rica, específicamente en lo que respecta al inciso b del artículo 4, pues debilita la participación de la Escuela de Formación Docente. Cabe señalar que desde esta normativa se administran las carreras de educación secundaria de la Universidad de Costa Rica.

Esta Resolución mantiene el Comité de las Carreras Compartidas integrado por los catorce directores y directoras de las Unidades Académicas que participan en la formación de docentes de educación secundaria: Formación Docente, Artes Plásticas; Artes Musicales; Biología; Filosofía; Filología, Lingüística y Literatura; Física; Geografía, Geología, Historia; Lenguas Modernas; Matemática; Psicología y Química; sin embargo, incluye un cambio, el cual es que ya no será presidida por la persona que ocupe la dirección de la Escuela de Formación Docente, sino que la presidirá una persona miembro que se designe en su seno, por votación, por un plazo de dos años y reelegible por una única vez.

Como se evidencia, la Resolución VD-R-8873-2012 (UCR, 2012c), emitida por el Dr. Bernal Herrera, vicerrector de Docencia, regula la formación de docentes en la Universidad de Costa Rica, disminuye la participación de la Escuela de Formación Docente de la Facultad de Educación y fortalece a las Unidades Académicas disciplinares.

El 7 de octubre del 2016, el Dr. Bernal Herrera, vicerrector de Docencia, firma la Resolución VDR-9454-2016 (UCR, 2016) creando la carrera de Bachillerato y Licenciatura en Educación Matemática, como responsabilidad exclusiva de la Escuela de Matemática, plan de estudio tecnocrático, disciplinar con influencia del pensamiento neoliberal que incluye cursos didácticas específicas; pero no de pedagogía y presenta debilidades de acuerdo con la teoría y la investigación mundial más reciente sobre formación docente (Murillo, 2006; Pogre, P.y Krichesky, G., 2005; Robalino, 2009; UNESCO-OREALC, 2014; Villega-Reimers, 2012), y en contraposición de la normativa nacional y universitaria: Ley Fundamental de Educación, Estatuto Orgánico (Artículo 3, Artículo 4 incisos b, d y f; Artículo 200), Principio de Departamentalización en Acta del Consejo Universitario No 2728 del 16 de setiembre de 1980 y N. 2762 del 2 de marzo 1981(UCR, 1980; UCR, 1981), Políticas Universitaria 2016-2020 (UCR,2015a), Directrices del 
Centro de Evaluación Académica para la creación de planes de estudio (UCR, 2015b). Este plan de estudio se pretende ofrecer en el curso lectivo 2017. Ante esta situación, la directora de la Escuela de Formación Docente presenta un recurso de adición y aclaración a la Vicerrectoría de Docencia.

La Escuela de Formación Docente, en la actualidad, se encuentra integrada en tres Departamentos: Educación Preescolar y Primaria, Educación Secundaria y Docencia Universitaria, de estos, al Departamento de Educación Secundaria le corresponde administrar y brindar los cursos correspondientes al núcleo pedagógico o área de formación pedagógica, en coordinación con otras Unidades Académica, a saber, Escuela de Orientación y Educación Especial y Escuela de Administración Educativa. Cabe señalar que el área de formación pedagógica no solo se brinda a los bachilleratos en la enseñanza secundaria, sino también a las carreras de Preescolar, Primaria, Bibliotecología y Educación Física. En la organización curricular del Departamento de Educación Secundaria, se distinguen cuatro áreas de formación académica:

Área de Fundamentos Educativos

Área de Didácticas específicas

Área de Investigación Educativa

Área de Formación Complementaria

Estas áreas se visualizan, se vivencian y se experimentan en los cursos que integran la formación pedagógica. Las carreras de educación secundaria que se ofrecen en el año 2016 son las siguientes:

- Bachillerato en la Enseñanza de las Ciencias Naturales y Licenciatura en la Enseñanza de la Química, la Física y la Biología

- Bachillerato en la Enseñanza del Inglés

- Bachillerato en la Enseñanza del Francés

- Bachillerato en la Enseñanza de la Filosofía

- Bachillerato en la Enseñanza de la Psicología

- Bachillerato en la Enseñanza de las Artes Plásticas

- Bachillerato y Licenciatura en la Enseñanza de la Matemática

- Bachillerato y Licenciatura en la Enseñanza de los Estudios Sociales y Educación Cívica

- Bachillerato y Licenciatura en la Enseñanza de la Música

- Bachillerato y Licenciatura en la Enseñanza del Castellano y la Literatura

El Bachillerato en la Enseñanza de la Educación Física o Ciencias del Movimiento Humano es administrado por Escuela de Educación Física de la Facultad de Educación.

El modelo de formación docente en la educación secundaria de la UCR es concurrente, lo que implica que la formación pedagógica y disciplinar se ofrecen simultáneamente, es decir, las personas que desean ser docentes no se forman primero en una disciplina y posteriormente en pedagogía, que es el modelo consecutivo. Sin embargo, en la UCR, el modelo concurrente no se lleva a cabo como en otros países, puesto que las Unidades Académicas de las disciplinas de la especialidad no ofrecen cursos específicos para el estudiantado de educación, al integrar la disciplina con la didáctica específica, sino que son cursos de contenidos de la disciplina; a pesar de que, desde siempre, la Escuela de Formación Docente ha insistido a las otras UA en la importancia de que la formación se dé bajo el modelo concurrente.

Para ingresar a la UCR como a cualquiera de las otras universidades públicas (UNA, ITCR, UNED), las personas interesadas deben aprobar una "prueba de aptitud académica" o examen de admisión general. Para seguir estudios universitarios en educación no se aplican pruebas específicas, basta con aprobar el examen de admisión y obtener la cantidad de puntos que exige el ingreso a las respectivas carreras, excepto los Bachilleratos en Educación Musical y Enseñanza de las Artes Plásticas, en las que las Escuelas de Música y Artes Plásticas solicitan una prueba de habilidad específica. A las universidades privadas se ingresa con la aprobación del Bachillerato en Educación Secundaria. 


\section{Planes de estudio: Bachillerato en la Educación Secundaria}

Estos bachilleratos tienen una duración de 8 semestres ( 4 años). La formación incluye las siguientes áreas:

\section{- "Área de cultura general humanística}

La visión humanista que poseen los planes de estudios es fundamental, ya que responde al planteamiento filosófico de la Universidad de Costa Rica, el cual consiste en inspirar y desarrollar el interés permanente por la cultura general y humanística, así como crear una conciencia crítica por la problemática costarricense, latinoamericana y mundial (Estatuto Orgánico de la Universidad de Costa Rica 2000).

Acorde con esta visión de humanismo, esta área la conforman los siguientes cursos: Humanidades, actividad deportiva y artística, repertorios, Seminarios de Realidad Nacional I y II, así como el Trabajo Comunal Universitario de 300 horas de trabajo debidamente planificado y sistematizado, que cada estudiante debe brindar al país sin remuneración alguna.

La formación humanista, según Rodrigo Facio, está inspirada por un ideal pedagógico que no es tanto enseñar, como enseñar a aprender, no es tanto decir lo que hay que pensar, como inducir a pensar. Formar un técnico sobre el hombre de ciencia y el hombre de ciencia sobre el hombre culto, moral y socialmente responsable. Con esta fundamentación filosófica, la Escuela de Estudios Generales de la Universidad de Costa Rica, contribuye en la formación humanista del futuro educador permitiéndole participar en la educación costarricense, desde una perspectiva holística" (Castro, 2004, p.16).

- Área de formación pedagógica

Esta formación es ofrecida por la Facultad de Educación mediante las siguientes Escuelas: Formación Docente (brinda de 28 a 34 créditos), Orientación y Educación Especial (9 créditos) y Administración Educativa (3 créditos), de la Facultad de Educación. El núcleo pedagógico comprende los siguientes cursos: Introducción a la Pedagogía, Desarrollo y Aprendizaje del Adolescente, Fundamentos de Didáctica, Principios de Currículum, Taller de Materiales Didácticos y Medios Audiovisuales, Principios de Evaluación y Medición Educativa, Metodología de la Enseñanza, Experiencia Docente, Psicopedagogía del Adolescente, Investigación para el Mejoramiento del Aprendizaje, Teoría de la Educación, Seminario de la Enseñanza. La mayoría de los cursos que se mencionan incluyen observaciones, experiencias didácticas e investigaciones acorde con los objetivos y la naturaleza de los cursos, estas acciones se desarrollan en centros educativos de educación secundaria.

Los cursos: Metodología de la Enseñanza, Experiencia Docente, y el Seminario de la Enseñanza se ofrecen de manera específica para cada uno de los énfasis o de formación. En la Tabla 1 se muestra la organización curricular del Núcleo Pedagógico, por Unidades Académicas que ofrecen los respectivos cursos, así como créditos, ciclos en que se ofrecen, incluyendo la salida lateral al Profesorado y el Título de Bachillerato.

Los estudiantes que se matriculan en los Bachilleratos para la Educación Secundaria, también deben aprobar cursos complementarios u opcionales, o sea, materias que ofrece el Departamento de Educación Secundaria de la Escuela de Formación Docente, los cuales brindan una formación específica en temas que se consideran necesarios para la formación integral del profesional en docencia. Esos cursos son los siguientes: Educación Ambiental, Educación Sexual y La Comunicación y los Procesos de Enseñanza Aprendizaje. 
TABLA 1

Cursos del área pedagógica de Profesorado y Bachillerato en la Educación Secundaria

\begin{tabular}{|c|c|c|c|c|c|}
\hline \multirow{3}{*}{$\begin{array}{l}\text { Nombres de los cursos } \\
\text { que corresponden el } \\
\text { Núcleo Pedagógico }\end{array}$} & \multicolumn{3}{|c|}{ Unidades Académicas que los ofrecen } & \multirow{3}{*}{$\begin{array}{l}\text { Ciclo en } \\
\text { que se } \\
\text { ofrece }\end{array}$} & \multirow{3}{*}{$\begin{array}{l}\text { Salida } \\
\text { lateral }\end{array}$} \\
\hline & $\begin{array}{l}\text { Formación } \\
\text { Docente }\end{array}$ & $\begin{array}{c}\text { Orientación } \\
\text { y Educación } \\
\text { Especial }\end{array}$ & $\begin{array}{l}\text { Administración } \\
\text { Educativa }\end{array}$ & & \\
\hline & Créditos & Créditos & Créditos & & \\
\hline Introducción a la Pedagogia & 3 & & & I & \multirow{2}{*}{$\begin{array}{l}P \\
R\end{array}$} \\
\hline $\begin{array}{l}\text { Desarrollo y Aprendizaje } \\
\text { en la Adolescencia }\end{array}$ & & 3 & & II & \\
\hline Fundamentos de Didáctica & 3 & & & III & \multirow{7}{*}{$\begin{array}{l}E \\
S \\
O \\
R \\
D\end{array}$} \\
\hline Principios de Currículum & 3 & & & III & \\
\hline $\begin{array}{l}\text { Principios de Evaluación y } \\
\text { Medición Educativa }\end{array}$ & & 3 & & IV & \\
\hline \multirow{2}{*}{$\begin{array}{l}\text { Taller de materiales } \\
\text { didácticos y medios } \\
\text { audiovisuales }\end{array}$} & & & 3 & IV & \\
\hline & & & & & \\
\hline \multirow{2}{*}{$\begin{array}{c}\text { Metodologia de la } \\
\text { Enseñanza de .... (se } \\
\text { ofrece para cada área de } \\
\text { formación) }\end{array}$} & 3 & & & V & \\
\hline & & & & & \\
\hline $\begin{array}{l}\text { Experiencia Docente en ... } \\
\text { (se ofrece para cada área de } \\
\text { formación) }\end{array}$ & 6 & & & VI & $o$ \\
\hline $\begin{array}{l}\text { Psicopedagogía del } \\
\text { Adolescente }\end{array}$ & & 3 & & VII & \multirow{5}{*}{$\begin{array}{c}\text { BACHI } \\
\text { LLERATO }\end{array}$} \\
\hline \multirow{2}{*}{$\begin{array}{c}\text { Investigación para } \\
\text { el Mejoramiento del } \\
\text { Aprendizaje }\end{array}$} & & 3 & & VII & \\
\hline & & & & & \\
\hline Teoría de la Educación & 3 & & & VIII & \\
\hline $\begin{array}{l}\text { Seminario en la Enseñanza } \\
\text { de... (se ofrece para cada } \\
\text { area de formación) }\end{array}$ & 4 & & & VIII & \\
\hline
\end{tabular}

\section{- Área de formación disciplinar}

Corresponde a la formación que brindan las Unidades Académicas disciplinares con énfasis en los contenidos propios de las respectivas disciplinas (60 a 84 créditos del Plan de Estudio). La Escuela de Formación Docente recomienda a dichas Unidades que estos cursos sean ofrecidos por docentes que tengan especialidad en la didáctica específica; sin embargo, en la mayoría de los casos son ofrecidos por especialistas en la disciplina.

Para obtener el bachillerato universitario, la población estudiantil debe realizar 300 horas de Trabajo Comunal Universitario (TCU). Estos Planes de Estudio le ofrecen al estudiantado la opción de obtener el título de Profesorado en la Enseñanza una vez cumplido el VI Ciclo (104 créd.) y 150 horas de TCU, lo cual lo habilita para que pueda ejercer labores como docente. Para optar por el grado de Bachillerato debe cumplir con 150 horas de TCU adicionales.

En la Tabla 2 se muestran las áreas y el creditaje de cada una de las carreras de Bachillerato en la Enseñanza. 
TABLA 2

Créditos por área de formación de los Bachilleratos en la Enseñanza

\begin{tabular}{lcccc}
\hline & \multicolumn{4}{c}{ Créditos por área de formación } \\
\cline { 2 - 5 } $\begin{array}{l}\text { Bachillerato en la enseñanza } \\
\text { de... }\end{array}$ & Humanidades & $\begin{array}{c}\text { Núcleo } \\
\text { pedagógico }\end{array}$ & Disciplina (s) & Total \\
\hline Artes Plásticas & 21 & 46 & 75 & 142 \\
\hline Ciencias Naturales 1 & 21 & 40 & 81 & 142 \\
\hline Psicología & 21 & 40 & 65 & 126 \\
\hline Música & 21 & 40 & 81 & 142 \\
\hline Matemática 2 & 21 & 40 & 70 & 131 \\
\hline Castellano y Literatura & 21 & 40 & 75 & 136 \\
\hline Inglés & 21 & 40 & 81 & 142 \\
\hline Francés & 21 & 40 & 84 & 145 \\
\hline Filosofia & 21 & 43 & 60 & 124 \\
\hline Estudios Sociales y Educ Civ. 3 & 21 & 40 & 74 & 135 \\
\hline
\end{tabular}

Notas: http://www.ucr.ac.cr/estudiantes/carreras/ciencias-sociales.html

1. De los 81 créditos disciplinares 24 los ofrece la Escuela de Biología, 23 la Escuela de Física, 20 la Escuela de Física, 6 de la Escuela de Matemática, 4 de la Escuela de Geología y 4 opcionales de un curso de las Escuelas mencionadas

2. De los 70 créditos disciplinares 68 los ofrece la Escuela de Matemática y 4 la Escuela de Física.

3. De los 74 créditos disciplinares 24 los ofrece la Escuela de Geografía y 60 la Escuela de Historia.

\section{Plan de estudio de Licenciatura en la Enseñanza Secundaria}

Estos planes de estudio tienen una duración de 4 semestres, dos planificados para ofrecerse como cursos regulares y dos para realizar una tesis (trabajo final de graduación). Las Licenciaturas en la Enseñanza se ofrecen para las siguientes áreas de estudio: Matemática, Castellano y Literatura, Estudios Sociales y Cívica, Música y Física, Química y Biología. Estos planes están constituidos por las siguientes áreas:

- Pedagogía general y específica: 2 o 5 cursos (6 o 15 créditos) ofrecidos por la Escuela de Formación Docente (EFD). Más 3 créditos de un curso optativo en algunas licenciaturas.

- Investigación Educativa: dos cursos de Investigación que imparte la EFD ( 6 créditos). La licenciatura en la enseñanza de la Matemática solo requiere uno de estos cursos.

- Disciplina (s): Ofrecen 8 a 4 cursos de acuerdo con la especialidad, algunos programas de licenciatura ofrecen cursos opcionales para que cada estudiante seleccione lo que desea matricular. La mayoría de los cursos opcionales corresponden a las mismas disciplinas del énfasis de la licenciatura.

- Tesis: Durante los dos últimos semestres los estudiantes deben realizar un trabajo final de graduación.

En la Tabla 3 se muestran las áreas y los créditos que incluyen las licenciaturas en la enseñanza de... 
TABLA 3

Créditos por áreas de formación de las Licenciaturas en la Enseñanza de Secundaria

\begin{tabular}{|c|c|c|c|c|}
\hline \multirow{3}{*}{$\begin{array}{l}\text { Licenciatura en la enseñanza } \\
\text { de... }\end{array}$} & \multicolumn{4}{|c|}{ Unidades Acadèmica } \\
\hline & \multicolumn{3}{|c|}{ Formación Docente } & \multirow{2}{*}{$\begin{array}{c}\begin{array}{c}\text { Unidades } \\
\text { disciplinares }\end{array} \\
\text { Disciplina }\end{array}$} \\
\hline & Pedagogia & $\begin{array}{c}\text { Cursos } \\
\text { investigación }\end{array}$ & Tesis & \\
\hline Estudios Sociales 1 & 6 & 6 & 2 & 13 y 6 (opt) \\
\hline Matemática 2 & 18 & 3 & 2 & 15 y 5 (opt) \\
\hline Música 3 & 6 y 3 (opt) & 6 & 2 & 10 y 6 (opt) \\
\hline Castellano y Literatura 4 & 6 & 6 & 2 & 18 (opt) \\
\hline Física 5 & 9 y 3 (opt) & 6 & 2 & 14 \\
\hline Química 6 & 9 y 3 (opt) & 6 & 2 & 17 \\
\hline Biología 7 & $9 \mathrm{y} 6$ (opt) & 6 & 2 & 6 y $12(\mathrm{opt})$ \\
\hline
\end{tabular}

Notas: http://www.ucr.ac.cr/estudiantes/carreras/ciencias-sociales.html

1 De los 19 créditos disciplinares, 7 los ofrece la Escuela de Historia, 6 la Escuela de Geografía y 6 son de cursos optativos que se seleccionan entre cursos que imparten la Escuela de Ciencias Políticas, la Escuela de Historia o la Escuela de Sociología.

2 De los 20 créditos disciplinares, 5 son para que el estudiantado seleccione entre cursos optativos de la Escuela de Matemática. 3 De los 16 créditos de la disciplina, 6 son para que el estudiantado seleccione entre cursos optativos del Área de Instrumento Complementario (2) y 1 curso del Área de Artes Musicales. 4 De los 18 créditos disciplinares el estudiantado tiene la opción de seleccionar 6 créditos del Área de Estudios Clásicos, 6 del Área de Lengua y 6 del Área de Literatura.

5 De los 17 créditos disciplinares, 7 son de cursos de la Escuela de Física, 4 la Escuela de Matemática, 3 la Escuela de Geología. 6 De los 20 créditos disciplinares, 14 son de cursos de la Escuela de Química, 3 la Escuela de Geología y 3 de un curso optativo que el estudiantado seleccione de la Escuela de Formación Docente, de la Escuela de Biología o de la Escuela de Estadística.

7 De los 18 créditos disciplinares, 6 son de cursos de la Escuela de Biología, 12 de cursos optativos que ofrece la Escuela de Biología, la Escuela de Estadística o la Escuela de Informática.

En la Universidad de Costa Rica la formación de docentes en educación física no se administra mediante Comisiones Compartidas, por lo que presenta a continuación su estructura curricular:

\section{Bachillerato en Educación Física o Bachillerato en Ciencias del Movimiento Humano}

La formación de docentes en educación física se realiza en la Escuela de Educación Física de la Facultad de Educación, no se administra mediante una Comisión Compartida. Es una carrera reacreditada, de 133 créditos. En el proceso de mejoramiento para la acreditación y reacreditación de esta carrera cambia su nombre a Bachillerato en Ciencias del Movimiento Humano. Las áreas de formación son las siguientes:

- Humanidades: son responsabilidad de la Escuela de Estudios Generales (21 créditos). Incluye los Cursos Integrados de Humanidades I y II, Seminario de Realidad Nacional I y II, actividad deportiva, actividad artística y un repertorio.

- Pedagogía: incluye los cursos Introducción a la Pedagogía, Principios de Currículum, Fundamentos de didáctica, que ofrece la Escuela de Formación Docente. Para un total de 9 créditos.

- Didácticas específicas: cursos que ofrece la Escuela de Educación Física. 34 créditos.

- Disciplina: cursos de la disciplina los brinda la Escuela de Educación Física. 32 créditos.

- Biología: un curso de 4 créditos que brinda la Escuela de Biología.

- Medicina: 3 cursos para un total de 13 créditos que ofrece la Escuela de Medicina.

- Química: 1 curso de 2 créditos que imparte la Escuela de Química.

- Opcionales: 2 cursos de optativos generales y 4 cursos opcionales del área que ofrece la Escuela de Educación Física. 
Para obtener el título del bachillerato universitario los estudiantes deben realizar las 300 horas de Trabajo Comunal Universitario.

En Costa Rica, con el título de Profesorado en la Enseñanza de las diferentes disciplinas, se puede ingresar al mercado laboral, sea contratado por el Ministerio de Educación Pública o por instituciones privadas. Se otorga el profesorado cuando se han aprobado los 6 primeros semestres y 106 créditos del Plan de estudios. Sin embargo, el estudiantado puede optar por los siguientes Certificados Intermedios: Suficiencia y Aptitud Superior, que les brinda la oportunidad de ingresar al Ministerio de Educación y a otras dependencias, a laborar como interinos, lo que les permite acumular puntos en el Servicio Civil y para su carrera profesional.

La Facultad de Educación de la Universidad de Costa Rica, además de ofrecer las carreras de grado, también brinda formación en posgrado, cuenta con una Maestría Académica con varias especialidades: Evaluación Educativa, Docencia Universitaria, Orientación Laboral, Orientación Familiar; además ofrece las siguientes Maestrías Profesionales: Administración Educativa, Administración Universitaria, Gestión Jurídica de la Educación, Bibliotecología y Estudios de la Información con énfasis en Gerencia de la Información y Tecnologías de la Información, Planificación Curricular, Recreación, Ciencias del Movimiento Humano; además, dos doctorados: en Educación y en Ciencias del Movimiento Humano.

\section{REFLEXIONES FiNALES}

Con base en el propósito de este artículo se presentan las siguientes reflexiones:

- La formación inicial de docentes de educación secundaria en la UCR, después de la Reforma Universitaria de 1957, ofrece una formación integral con un enfoque humanista la cual se ha mantenido hasta la actualidad. No obstante, en el año 2016, la Vicerrectoría de Docencia certifica a la Escuela de Matemática para que asuma a partir del año 2017, las carreras de Bachillerato y Licenciatura en Educación Matemática. Cabe señalar que esta formación académica está constituida por un enfoque disciplinar tecnocrático contrario a la normativa nacional y universitaria, a lo que establece la teoría e investigación reciente sobre formación de docentes, y con influencia del pensamiento neoliberal. Esta carrera no incluye cursos de pedagogía, ni la participación de la Facultad de Educación en la formación del futuro profesorado.

- En relación con la formación que se ofrece en otros países (Cuba, Finlandia, Alemania, Singapur), la de la UCR coincide en las áreas de formación que contemplan nuestros planes de estudio, pues se ofrecen cursos de las áreas de: pedagogía, investigación, práctica docente y la (s) disciplina (s). En esta última área la diferencia radica en que, en los países mencionados, la(s) disciplina(s) se ofrece(n) simultáneamente con su didáctica, mediante cursos colegiados donde trabajan colaborativamente personal docente especialista en la disciplina con especialista en didáctica. Por otra parte, en Alemania, Cuba y Finlandia, profesionales en educación secundaria se forman de manera obligatoria en dos disciplinas, no en una como sucede en Costa Rica. Además, en los países citados, las observaciones y las prácticas se inician ingresando a la carrera y se enfatiza en la investigación educativa durante todo el proceso formativo.

- En Costa Rica, así como en el resto de los países estudiados, la formación de docentes se ofrece en la educación superior mediante universidades pedagógicas, institutos o centros de educación de docentes y facultades de educación cuyo objeto de estudio es la formación docente.

- Los planes de estudio en educación secundaria que se ofrecen en la actualidad tienen su inicio en 1991, en el transcurso del tiempo se le han realizado modificaciones relativas a cambios de nombres, siglas, contenidos, bibliografía y ubicación de cursos. Estos cambios son difíciles de hacer porque el modelo de gestión administrativa y curricular exige que toda transformación debe ser aprobada por las Unidades Académicas involucradas en la formación de docentes. De ahí que la propuesta de cambios 
en los cursos del núcleo pedagógico debe obtener la aprobación de alrededor de trece asambleas de Unidades Académicas, esto hace muy lentas las innovaciones que se desean implementar. Por lo que es importante repensar el modelo de gestión vigente.

- La gestión administrativa y curricular de las carreras de educación secundaria, como se indicó anteriormente, se convierte en una tarea compleja y, por consiguiente, los procesos de autoevaluación e innovación de esas carreras también se complican, de ahí que los esfuerzos que se han realizado para llevar a cabo esas tareas no han dado los frutos esperados.

- Las carreras de educación secundaria deben responder a las características, necesidades e intereses de la población que atienden (adolescentes, jóvenes y personas adultas), para responder a los requerimientos del contexto nacional e internacional, de ahí la necesidad de promover la autoevaluación constante de esas carreras para su actualización, mejoramiento y acreditación.

- La formación de profesionales en educación es una tarea de gran responsabilidad, por la delicada función que ejercen en la formación de una ciudadanía que contribuya al desarrollo humano, económico y sostenible en busca del bien común y la equidad social.

- La formación docente en la Universidad de Costa Rica evidencia una formación interdisciplinar. De acuerdo con la estructura curricular de los planes de estudio, el estudiantado debe matricular cursos de humanidades, actividad deportiva, actividad artística, realidad nacional, de pedagogía, teoría de la educación, currículo, psicología, didáctica general y específica, investigación, observaciones, prácticas docente, opcionales y del contenido disciplinar de la especialidad, estas últimas representan alrededor de un $60 \%$ de la formación. Una recomendación necesaria es que los cursos de las disciplinas se ofrezcan con sus didácticas específicas y en relación directa con la práctica en instituciones de educación secundaria, para que el futuro profesorado desarrolle las habilidades y destrezas requeridas para su desempeño profesional. Por lo tanto, en términos generales, se considera que la Universidad de Costa Rica, a través de las Unidades Académicas que asumen la formación académica de profesionales en educación, ofrece una eficiente formación humanística, disciplinar y pedagógica para el futuro profesorado. Por consiguiente, quienes desean estudiar cualquiera de las enseñanzas que ofrece esta Universidad logran un nivel académico elevado, que les permite ejercer exitosamente sus funciones docentes en los diferentes contextos educativos.

\section{ReFERENCIAS}

Asamblea Legislativa de la República de Costa Rica. (1957). Ley Fundamental de Educación N. 2160 . San José, Costa Rica.

Castro, J (julio-diciembre, 2004). La enseñanza de las artes plásticas en la Universidad de Costa. Revista Electrónica Actualidades Investigativas en Educación, 4(2), 1-20. Recuperado de http://www.redalyc.org/ $\mathrm{html} / 447 / 44740206 /$

Chaves, L. (2011). Informe de labores del Decanato de la Facultad de Educación. San José, Costa Rica. Manuscrito inédito.

Chaves, L. (2012a). Informe de labores del Decanato de la Facultad de Educación. San José, Costa Rica. Manuscrito inédito.

Chaves, L. (12 abril 2012b). Una Resolución de la Rectoría Institucionalmente Histórica. Periódico La Nación. Recuperado de http://www.nacion.com/opinion/foros/resolucion-Rectoria-institucionalmentehistorica_0_1262073930.html

Dengo, M. E. (2013). El desarrollo de la formación docente en Costa Rica. En J. Salazar (Ed.), Historia de la educación costarricense ( $2^{\mathrm{a}} \mathrm{ed}$.). San José, Costa Rica: EUNED.

González, Y. (2013). Educación diversificada y humanística para una democracia integral (1950-1970). En J. Salazar (Ed.), Historia de la educación costarricense ( $2^{a}$ ed.), San José, Costa Rica: EUNED. 
Ministerio de Educación Pública. (1958). Informe sobre el estado actual de los trabajos de reforma de la segunda enseñanza costarricense. San José, Costa Rica: Publicaciones MEP.

Murillo, J. (2006). La formación docente: Una clave para la mejora educativa. En Modelos innovadores de formación inicial docente. Una apuesta por el cambio. Santiago de Chile: UNESCO-PRELAC.

Pogre, P. y Krichesky, G. (2005). Formar docentes una alternativa multidisciplinaria. Argentina: Papers Editores.

Polanco, M. E. (1990). Profesores de segunda enseñanza y Reforma Universitaria de 1957. Revista Educación. 14(1), 37-57. Recuperado de https://docs.google.com/viewerng/viewer?url=http://revistas.ucr.ac.cr/index.php/ educacion/article/viewFile/18428/18598

Robalino, M. (2009). Políticas educativas, políticas docentes y formación docente. Quito: Oficina UNESCO.

Universidad de Costa Rica. (1960). Reglamento de la Escuela de Educación. San José, Costa Rica: Autor.

Universidad de Costa Rica. (1980). Acta del Consejo Universitario N. 2728 del 16 de setiembre de 1980. San José, Costa Rica: Autor.

Universidad de Costa Rica. (1981). Acta del Consejo Universitario N. 2762 del 2 de marzo 1981. San José, Costa Rica: Autor.

Universidad de Costa Rica. (1989). Resolución VD-R-4379-89. San José, Costa Rica: Autor.

Universidad de Costa Rica. (2000). Resolución Adición a la Resolución VD-R-4379-89. San José, Costa Rica: Autor.

Universidad de Costa Rica. (2012a). Resolución VD-R- 8782.2012 San José: Autor.

Universidad de Costa Rica. (2012b). Resolución R-2616-2012. San José: Autor.

Universidad de Costa Rica. (2012c). Resolución VD-R-8873-2012. San José: Autor.

Universidad de Costa Rica. (2015a). Políticas de la Universidad de Costa Rica 2016-2020 "Excelencia e Innovación con Transparencia y Equidad" Recuperado de http://www.cu.ucr.ac.cr/uploads/ tx_ucruniversitycouncildatabases/normative/politicas_institucionales_2016-2020.pdf

Universidad de Costa Rica. (2015b). Aspectos por considerar al elaborar propuestas de modificación parcial o integral de planes de estudio y para la creación de carreras. Normativa curricular asociada. San José: Centro de Evaluación Académica, Vicerrectoría de Docencia. Universidad de Costa Rica. Recuperada de http://www.cea.ucr.ac.cr/ media/diea/Modificaciones-a-planes-propues.pdf

Universidad de Costa Rica. (2016). Resolución VD-R-9454-2016. San José: Autor.

UNESCO-OREALC. (2014). Temas críticos para formular nuevas políticas docentes en América Latina y el Caribe: El debate actual. Santiago, Chile: Centro de Estudios de Políticas Educativas. Universidad Católica.

Villegas-Reimers, E. (2012). Desarrollo profesional docente. Heredia: Universidad Nacional.

\section{BY-NC-ND}

